\title{
Variações na forma do otólito sagitta de Coryphaena hippurus (Actinopterygii: Coryphaenidae) em uma área de ressurgência na costa sudoeste do Oceano Atlântico
}

\author{
Paulo R. C. Almeida' (D), Cassiano Monteiro-Neto' (D), Rafael A. Tubino² (D) \& Marcus R. Costa ${ }^{1}$ (D)
}

\footnotetext{
1. Departamento de Biologia Marinha, Universidade Federal Fluminense, Caixa Postal 100644, 24001-970 Niterói, RJ, Brasil. (prcalmeida@id.uff.br; cmneto@id.uff.br; marcusrc@id.uff.br)

2. Departamento de Biologia Animal, Universidade Federal Rural do Rio de Janeiro, Rodovia BR-465, Km 07, 23890-000, Seropédica, RJ, Brasil. (rattubino@gmail.com)
}

Recebido 21 fevereiro 2019

Aceito 29 junho 2020

Publicado 07 agosto 2020

DOI 10.1590/1678-4766e2020019

\begin{abstract}
Variations in the shape of the sagitta otolith of Coryphaena hippurus (Actinopterygii: Coryphaenidae) in a resurgence area on the southwest coast of the Atlantic Ocean. The otoliths are structures species-specific of fishes whose morphologic analysis is an efficient tool for identification and discrimination of stocks and/or populations subjected to clinal variations. This work intends to show the degrees of morphologic variation in sagitta otoliths of dolphinfish, Coryphaena hippurus Linnaeus, 1758, landed by the fishing fleet from Rio de Janeiro. The 164 pairs of otoliths analyzed were obtained from biological samples collected between February 2014 and October 2016. The shape and the contour of sagitta otoliths were characterized by means of six descriptors (area, perimeter, circularity, rectangularity, eccentricity and fractal dimension) and Elliptical Fourier Analysis (EFA), from the images of otoliths. Interannual comparisons of shape descriptors showed significant differences (PERMANOVA p $<0.0001$ ) except rectangularity and fractal dimension. The linear discriminant analysis (LDA) between years exhibited a better classification (94.8\%) for 2015, when the data was more consistent. The cluster analysis of the 30 harmonics based on Ward's hierarchical algorithm revealed four otolith morphotypes. The principal components analysis (PCA) from harmonics of the 82 otoliths examined did not show spatial discrimination between fishing areas. Therefore, the observed morphotypes corroborate the existence of a polymorphism in the study area, determined by balanced temporal selection. This supposition can be supported by genetic studies realized in the region, where the hypothesis of two commercial morphotypes was rejected, confirming the presence of a mixed stock.
\end{abstract}

KEYWORDS. Geometric morphometrics, otolith shape, elliptic Fourier analysis, multivariate analysis.

RESUMO. Os otólitos são estruturas espécie-específicas de peixes cuja análise morfológica é uma ferramenta eficiente para identificação e discriminação de estoques e/ou populações sujeitas a variações clinais. O presente trabalho buscou elucidar o grau de variação morfológica dos otólitos sagitta do dourado, Coryphaena hippurus Linnaeus, 1758 capturado ao largo da costa sudeste do Brasil pela frota pesqueira sediada no Rio de Janeiro. Os 164 pares de otólitos analisados foram obtidos a partir de amostras coletadas entre fevereiro de 2014 e outubro de 2016 . A forma e o contorno dos otólitos sagitta foram caracterizados através de seis descritores (área, perímetro, circularidade, retangularidade, excentricidade e dimensão fractal) e análises de Fourier elíptica (AFE), a partir de imagens dos otólitos. Comparações interanuais dos descritores de formas apontaram diferenças significativas (PERMANOVA $\mathrm{p}<0,0001)$, com exceção da retangularidade e dimensão fractal. A análise discriminante linear (ADL) entre anos, apresentou melhor classificação (94,8\%) para o ano de 2015, cuja disponibilidade de dados foi mais consistente. A análise de agrupamento das 30 harmônicas, baseada no algoritmo hierárquico de Ward, revelou quatro morfotipos de otólitos. A análise de componentes principais (ACP) das harmônicas dos 82 otólitos examinados não discriminou as áreas de captura. Assim, os morfotipos observados denotam a existência de um polimorfismo em toda área de estudo, determinado provavelmente por um balanço de seleção, i.e., seleção por equilíbrio temporal. Tal suposição foi corroborada através de estudos genéticos realizados nesta região, cuja hipótese da presença de dois morfotipos capturáveis/comercializáveis (dourado vs. palombeta) na área foi rejeitada, confirmando a presença de um estoque misto.

PALAVRAS-CHAVE. Morfometria geométrica, forma do otólito, análise de Fourier elíptica, análise multivariada.

Os otólitos são estruturas policristalinas compostas basicamente de carbonato de cálcio em forma de aragonita, outros minerais em menores proporções e uma matriz proteica, configurando componentes essenciais do sistema de audição e equilíbrio de peixes ósseos (CAMPANA, 1999; POPPER \& Lu, 2000). Os padrões de crescimento e composição química estão relacionados com o hábito e história de vida das espécies, bem como com o ambiente, constituindo uma importante fonte de informações acerca dos indivíduos (CAMPANA et al., 2000; Cardinale et al., 2004; Baumann et al., 2006). Neste contexto, análises de forma de otólitos, realizadas a partir de métodos geométricos, constituem uma abordagem prática e eficiente para determinar e distinguir estoques pesqueiros, essencial na aplicação de estratégias de manejo de espécies e ecossistemas (CAMPANA \& CASSELMAN, 1993).

Um estoque pesqueiro pode ser definido como conjunto de indivíduos de uma espécie sob processo de explotação em determinada região, apresentando características locais intrínsecas como interação com o ambiente e parâmetros de história de vida (Sparre \& Venema, 1997; BegG \& 
WaLdman, 1999). A importância de se definir os limites de um estoque, conhecer sua dinâmica e história de vida reside no fato de esse conhecimento permitir a aplicação de estratégias de manejo que visem otimizar a explotação do recurso e mantê-lo disponível perpetuamente (SPARRE \& Venema, 1997; VaZ-Dos-Santos et al., 2007). Para tal, são necessárias pesquisas científicas com objetivo de entender o comportamento das espécies perante as condições ambientais e de explotação, bem como acompanhar permanentemente, a médio e longo prazo, de modo a aplicar ações emergenciais quando necessário. Estudos morfométricos e morfológicos de otólitos têm sido utilizados na identificação de várias espécies marinhas de importância ecológico-econômica em diversas regiões (CASTONGUAY et al., 1991; CAMPANa \& CASSELMan, 1993; TUSET et al., 2003; MAHE et al., 2016), inclusive ao largo da costa do Brasil (Monteiro et al., 2005; RoNDON et al., 2014; SANTOS et al., 2017; SoETH et al., 2019).

Nas últimas décadas, estudos em diferentes escalas (mundiais, regionais e locais) têm demonstrado que as alterações climáticas e o monitoramento das áreas de ressurgência estão entre os fenômenos mais estudados, tendo como objetivo compreender a variabilidade das populações naturais nos diferentes ecossistemas. Flutuações em índices que mensuram tais variabilidades, i.e, NAO North Atlantic Oscilation, AMO - Atlantic Multidecadal Oscilation, ENSO - El Nino Southern Oscilation, bem como em áreas de ressurgência entre $10^{\circ}$ e $30^{\circ}$ de latitude, que estão entre as regiões mais produtivas do planeta (costas do Peru, Califórnia, Canárias, Benguela e Cabo Frio) (Ryther, 1969), têm sido relacionadas com flutuações em populações de peixes, incluindo os pequenos e grandes pelágicos (PAULY \& Christensen, 1995). Na costa Brasil, durante o verão, as águas quentes e salinas da Corrente do Brasil influenciam águas situadas mais ao sul da plataforma continental do sudeste-PCSE, elevando a temperatura da superfície do mar (TSM) da região (STEVENSON et al., 1998). Já no outono e no inverno, a maior influência vem das águas frias oriundas das altas latitudes, gerando um resfriamento da água sobre a plataforma interna sudeste e sul do Brasil. Ainda durante o inverno, há um enfraquecimento da Corrente do Brasil, possibilitando uma grande intrusão de águas frias vindas do Sul, chegando a influenciar as águas da Plataforma Continental do Sudeste do Brasil (PCSE). Já no verão, a massa de água que aflora ao largo da porção norte da PCSE - região de Cabo Frio é a Água Central do Atlântico Sul (ACAS), é fria e com elevadas concentrações de nutrientes. Nesta região ocorrem importantes concentrações de peixes pelágicos como sardinhas, atuns e afins (MUEHE \& GARCEZ, 2005).

O dourado Coryphaena hippurus Linnaeus, 1758 (Actinopterygii: Coryphaenidae) é um dos mais importantes recursos pelágicos do Atlântico Sudoeste (Ávila-DA-Silva \& VaZ-Dos-Santos, 2000; Dallagnolo \& ANDRADE, 2008), sendo intensamente capturado pelas frotas comerciais e pescadores esportivos. É um predador de topo de cadeia alimentar, com ampla distribuição geográfica e comportamento migratório (PALKO et al., 1982). Apresenta atributos típicos de espécie " $r$ " estrategista, tais como distribuição circuntropical, hábitos pelágicos, crescimento rápido e desova sincronizada quando o alimento é abundante (OXENFORD, 1999). Até o momento, apenas um estudo foi realizado ao largo do atlântico ocidental envolvendo métodos de separação de estoques de dourado com base na morfologia de otólitos, o qual separou dois estoques no nordeste do Brasil (DuARTE-Neto et al., 2008). Tal circunstância aponta para necessidade de aprofundamento dos estudos envolvendo a dinâmica de estoques da espécie, que representa um importante recurso pesqueiro compartilhado e que demanda estratégias de manejo específicas de acordo com os distintos níveis de explotação (PALKo et al., 1982; Sparre \& Venema, 1997). Nosso objetivo foi descrever a morfologia do otólito e identificar eventuais diferenças na forma dos otólitos em uma ecorregião de ressurgência costeira. Para tal, foram formuladas as seguintes questões: a) as técnicas morfométricas e morfológicas aplicadas são capazes de identificar distintas unidades de estoques? b) as áreas de pesca propiciam a separação de distintos morfotipos? Assim, esperamos verificar se os atributos geomorfológicos e o processo de ressurgência que ocorre na área de pesca apresentam alguma relação dedutiva com a forma dos otólitos da espécie, resultando em atributos populacionais idênticos ou desiguais. Logo, a variabilidade intraespecífica na forma dos otólitos pode indicar isolamento geográfico com atributos típicos de distintas unidades de estoque, ou a presença de um estoque misto com características genéticas semelhantes e traços fenotípicos variáveis em função da condição dos indivíduos e/ou do meio.

\section{MATERIAIS E MÉTODOS}

Área de estudo. A área de estudo compreende a porção norte da baía do Sudeste, desde Ilha Bela (SP) até o litoral Norte fluminense (RJ) (Fig. 1), integrando parte da PCSE, cuja linha de costa neste domínio geográfico é irregular com aproximadamente $600 \mathrm{~km}$. De uma forma geral, as isóbatas nessa região estão dispostas paralelamente à linha de costa e evidenciam um tênue declive em direção à quebra da Plataforma Continental (PC). Segundo CASTRO \& MIRANDA (1998), a porção Norte da PCSE pode ser dividida em distintas subáreas de acordo com a sua morfologia. A subárea mais ao Norte, que se estende deste Arraial do Cabo (RJ) até o Cabo de São Tomé (RJ) e é caracterizada por apresentar a linha de costa orientada de SW/NE, PC mais extensa com largura desde a linha de costa até a quebra da PC, variando de 70 a $100 \mathrm{~km}$ e a profundidade de quebra de PC mais rasa, entre 80 e 100 metros. A subárea central, que vai de Arraial do Cabo (RJ) até Ilha Grande (RJ) é definida pela abrupta mudança na orientação da linha de costa, que passa a ser de E/W. Esta última subárea também é caracterizada por possuir uma PC mais estreita próximo a Arraial do Cabo (RJ), alargando-se conforme o aumento da latitude e variando de 50 a $150 \mathrm{~km}$ de extensão. A profundidade de quebra de PC é comparativamente maior, em torno de 150 a $200 \mathrm{~m}$. A subárea mais ao sul, que vai de Paraty (RJ) até Ilhabela (SP) 


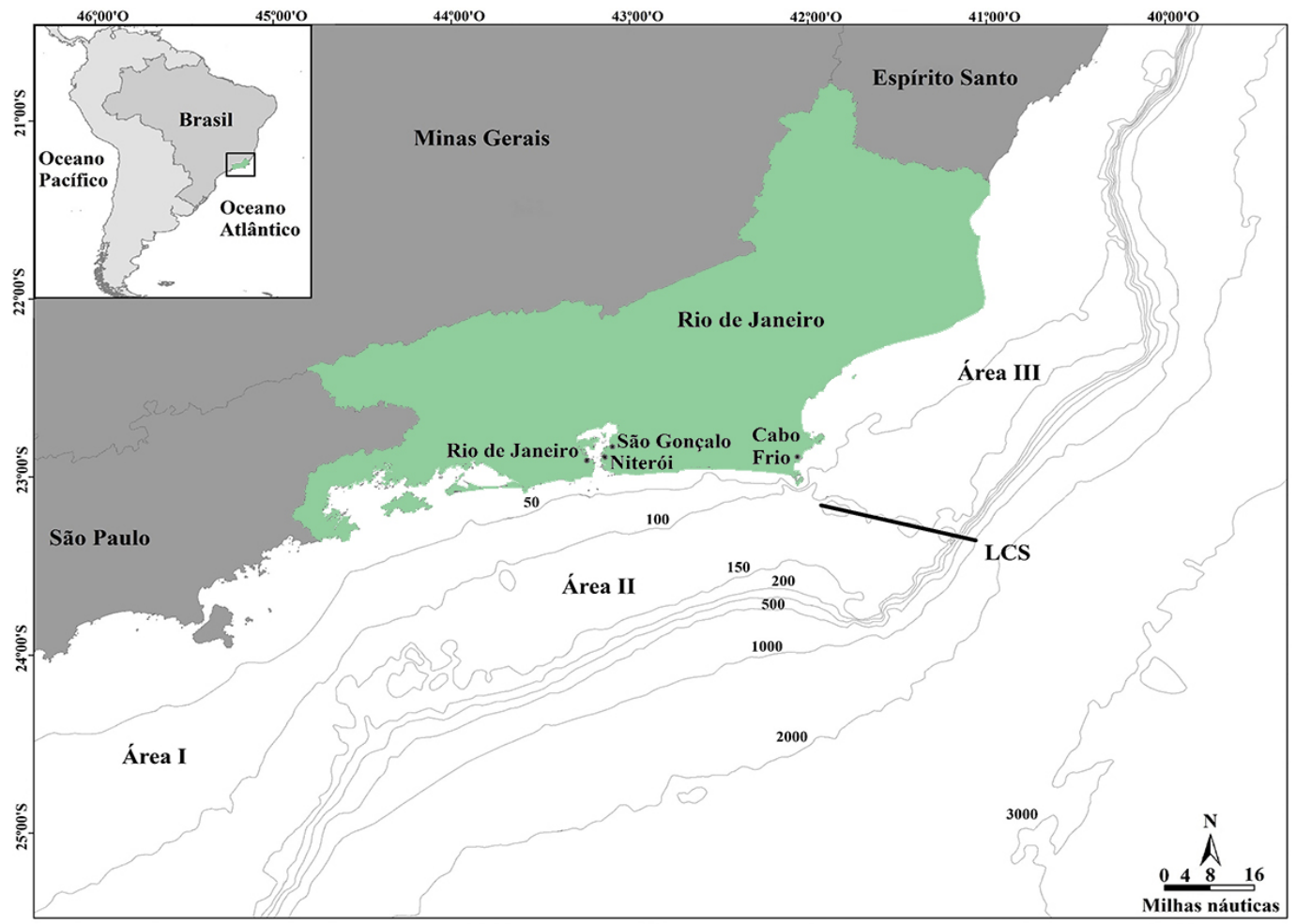

Fig. 1. Região de estudo no Sudoeste do Uceano Atlântico, com indicação das três áreas de atividade da frota pesqueira que captura C'oryphaena hippurus Linnaeus, 1758 desembarcado nos portos do Rio de Janeiro, Brasil (LCS, Lineamento Cruzeiro do Sul, indicando alteração no sentido da plataforma continental).

apresenta linha de costa caracterizada pela orientação SW/ NE. A plataforma na região apresenta carácter irregular, com um aumento da declividade na plataforma interna, próximo à ilha de São Sebastião (SP). As isóbatas se distribuem apresentando estreitamento e aumento da declividade na plataforma, sobretudo entre as profundidades de 80 e 110 metros.

A hidrodinâmica marinha nesta região da PCSE sofre a influência de distintas forçantes: a componente atmosférica, a Corrente do Brasil (CB), a Maré e um processo oceanográfico de larga escala - ressurgência - que afeta a estrutura e dinâmica do ecossistema pelágico em toda PCSE. O processo de ressurgência é desencadeado principalmente em consequência dos ventos NE, transporte de Ekman das águas de superfície e geomorfologia local, fertilizando a zona eufótica (Silva et al., 1988; VAlEnTin \& CoutinHo, 1990) com efeitos até $400 \mathrm{~km}$ a sudoeste sobre a plataforma de São Paulo (LoREnZZETti \& GAETA, 1996). A ocorrência de meandros e vórtices ciclônicos e anticiclônicos, eventos que induzem ressurgência de quebra de plataforma, também é relatada em toda a amplitude da região de estudo, cuja ocorrência se deve às mudanças de orientação da linha de costa e ao gradiente da topografia de fundo (CASTRO \& LEE, 1995).

As áreas de atuação das frotas que capturam $C$. hippurus ao largo da porção norte da PCSE são: Área I (litoral norte de São Paulo), Área II (costa sul fluminense) e Área III (costa norte fluminense) (Fig. 1). Separando as áreas II e III se encontra o lineamento Cruzeiro do Sul, que separa as bacias de Santos e Campos (MoHriak et al., 2010).
Coleta dos dados. Entre fevereiro de 2014 e outubro de 2016, exemplares de C. hippurus foram obtidos aleatoriamente de distintas capturas comerciais (linha de mão, espinhel de superfície e emalhe) desembarcadas em alguns dos principais portos de descarga da frota comercial localizados nas cidades de Niterói e Cabo Frio (RJ). Imediatamente após a coleta, os peixes foram preservados em gelo para posteriormente serem processados no Laboratório de Biologia do Nécton e Ecologia Pesqueira da Universidade Federal Fluminense. Todos os indivíduos foram medidos quanto ao seu comprimento furcal $(\mathrm{CF}, \mathrm{em} \mathrm{cm})$, pesados (peso total, PT, em g) e identificados quanto ao sexo. Os 164 pares de otólitos sagitta foram extraídos através de uma abertura na região dorsal do neurocrânio dos exemplares. Após a retirada, os mesmos foram lavados e armazenados a seco.

Para realização das análises morfométricas, todos os indivíduos foram utilizados, enquanto que para a Análise de Fourier Elíptica (AFE), foram selecionados apenas 82 indivíduos capturados no ano de 2015, provenientes das capturas de espinhel de superfície com CF variando entre 55 e $75 \mathrm{~cm}$ (todos adultos) e com probabilidade de idade semelhante, evitando assim efeitos de variações ontogenéticas na forma dos otólitos. Os otólitos foram orientados horizontalmente com o rostro e antirostro para a esquerda e com sulco para cima, sendo fotografados com auxílio de um microscópio estereoscópio com câmera acoplada modelo Zeiss Axiocam ERc5s, com luz refletida sobre um fundo preto e escala em micrômetros. As imagens foram processadas posteriormente no software ImageJ. 
Descritores de forma. Os descritores de forma utilizados para caracterizar os otólitos foram a área (A) e o perímetro (P), obtidos através do software ImageJ, enquanto que os índices de circularidade, retangularidade e excentricidade foram calculados de acordo com metodologia proposta por TUSET et al. (2003). A dimensão fractal foi calculada utilizando o plugin FracLac, do software ImageJ, através do método de contagem de caixas. A circularidade estima a similaridade de uma estrutura em relação a um círculo perfeito utilizando um valor mínimo de $4 \pi(12,57)$. A retangularidade descreve as variações do comprimento e da largura em relação a uma determinada área, onde o valor 1 , representa um retângulo perfeito. Já a excentricidade determina a posição do centro de massa em relação ao de um círculo perfeito (Russ, 1990). A dimensão fractal foi utilizada devido ao seu amplo uso na representação de formas e retrata fenômenos naturais com padrões recorrentes. Ela não permite a comparação de contornos com relação à forma, mas sim, quanto a sua complexidade, i.e., maiores valores correspondem a maior complexidade numa escala entre 1 e 2 (MonteIro \& ReIs, 1999; Costa \& Cesar JR, 2010). Os coeficientes de Fourier (FC) foram calculados utilizando análise de Fourier elíptica (AFE) geradas pelo software SHAPE (IWATA \& UKAI, 2002), com base nos eixos dos componentes principais e séries de Fourier denominadas harmônicas. O método representa um ajuste de funções das harmônicas em relação ao contorno original do otólito com uma elipse como o primeiro passo de aproximação, decompondo as variações das coordenadas $\mathrm{x}$ e y separadamente, como funções paramétricas da distância ao longo do contorno (KUHL \& Giardina, 1982).

Análises estatísticas. Todos os descritores de forma foram testados quanto à normalidade e homogeneidade de variâncias, sendo, então, log-transformados para realização das análises estatísticas, com exceção dos FCs. Como as premissas de homogeneidade e homocedasticidade não foram atingidas para todos os descritores de forma, foram aplicados apenas testes não-paramétricos. Testes $U$ de Mann Whitney conjugados a análises de Monte Carlo baseados em 10.000 atribuições aleatórias foram aplicados para avaliar diferenças significativas no comprimento do otólito (diâmetro de Feret) entre os exemplares direito e esquerdo, bem como entre os sexos (CALLICó-ForTunATO et al., 2017). Como a hipótese nula foi satisfeita, os sexos foram agrupados e o otólito esquerdo utilizado para as análises e nos casos em que este estava danificado o direito foi usado. O efeito do comprimento do peixe e do otólito (diâmetro de Feret) em relação a cada descritor de forma foi avaliado através de análises das inclinações de regressões lineares através de teste $F$, com exceção dos coeficientes de Fourier, os quais foram interpretados como invariantes em relação ao tamanho (DiAz et al., 1997). Os descritores que apresentaram correlação significativa com o comprimento do peixe foram corrigidos utilizando o coeficiente angular " $b$ " das equações (LleONART et al., 2000). Para comparações entre os descritores de forma por áreas de pesca e ciclos anuais foram realizadas análises multivariadas permutacionais de variância (PERMANOVA), baseadas em matrizes de similaridade utilizando a distância euclidiana (ClaRKE \& Gorley, 2006). Sempre que a hipótese nula foi rejeitada, um teste a posteriori (pairwise-test) foi aplicado, a fim de identificar diferenças significativas (ANDERSON et al., 2008). Um teste de homogeneidade de matrizes de covariância foi utilizado para verificar qual tipo de análise discriminante aplicar. Como a hipótese nula foi satisfeita, uma análise discriminante linear (ADL) foi utilizada para avaliar os padrões de diferenciações morfométricas entre os ciclos anuais, cujo sucesso de classificação foi determinado a partir do método Jackknife, em que a função é recalculada várias vezes, sempre deixando um dos indivíduos de fora da amostra e predizendo a sua identificação (VENABLES \& RIPLEY, 2002). Para determinação dos padrões de forma a partir dos coeficientes de Fourier, sem o estabelecimento de grupos prévios por área de captura, foram empregadas duas técnicas multivariadas, utilizando a matriz de dados composta pelas 30 primeiras harmônicas dos coeficientes de Fourier. Uma análise de agrupamento baseada no método de Ward (ReIs, 1997), onde a distância euclidiana foi utilizada como índice de similaridade entre os otólitos. E uma análise de componentes principais (ACP), cujo objetivo é reduzir a dimensionalidade dos dados, permitindo a caracterização da variabilidade entre grupos (áreas de pesca e possíveis morfotipos observados). Todas as análises foram realizadas utilizando os softwares $\mathrm{R}$ (análises univariadas, discriminantes e de agrupamento), SHAPE version 1.2 (análises de Fourier e de componentes principais) e PERMANOVA+ versão 1.0.2 (análises permutacionais univariadas e multivariadas).

\section{RESULTADOS}

Foram analisados um total de 164 indivíduos cuja amplitude de CF variou de 38,0 a $116,0 \mathrm{~cm}$, com valores médios mínimos e máximos de 52,0 e $81,9 \mathrm{~cm}$ (Tab. I). Enquanto os anos de 2014 e 2016 apresentaram menor abundância ( $n=23$ e 26, respectivamente), 2015 apresentou abundância elevada $(\mathrm{n}=115)$, cujas amostras foram subdividas entre as áreas de pesca para comparações a posteriori das medidas dos otólitos, em que os valores médios de $\mathrm{CF}$ variaram entre 61,4 e $65,8 \mathrm{~cm}$.

A circularidade foi a única variável a apresentar correlação com o comprimento do otólito de acordo com os testes de inclinação, sendo corrigida a partir do coeficiente angular da análise de regressão entre o comprimento do otólito e a variável.

Todos os descritores de forma analisados em conjunto apresentaram diferenças significavas entre os ciclos anuais (PERMANOVA, pseudo-F $=27,054 ; p<0,0001$ ). Quando avaliados isoladamente, quatro descritores de forma apresentaram diferenças significativas entre os anos, área, perímetro, excentricidade $(p<0,0001)$ e circularidade $(p<0,01)$ (Tab. II). O teste de comparações a posteriori (pairwise), aplicado aos quatro descritores com diferenças significativas, apresentou valores de $p$ variando entre 0,01 e 0,0001 e corroboraram as diferenças interanuais. 
Tab. I. Número de espécimes, por ano e área em 2015, amplitude, média e desvio padrão (DP) do comprimento furcal (CF), de Coryphaena hippurus Linnaeus, 1758 capturados na costa do Rio de Janeiro, Brasil.

\begin{tabular}{lcccc}
\hline Ano & $\mathrm{N}^{\circ}$ de otólitos & Amplitude $(\mathrm{cm})$ & Média \pm DP $(\mathrm{cm})$ \\
\hline \multicolumn{2}{c}{2014} & 23 & $46,8-112,0$ & $81,9 \pm 23,9$ \\
& 2015 & 115 & $45,0-116,0$ & $64,7 \pm 12,8$ \\
Área I & 24 & $47,9-115,0$ & $65,8 \pm 21,2$ \\
Área II & 53 & $51,0-116,0$ & $65,8 \pm 10,4$ \\
Área III & 38 & $45,0-74,8$ & $61,4 \pm 7,4$ \\
& 2016 & 26 & $38,0-72,5$ & $52,0 \pm 10,9$ \\
\multicolumn{2}{l}{ Total } & 164 & $38,0-116,0$ & $65,1 \pm 16,7$ \\
\hline
\end{tabular}

Tab. II. Comparação interanual dos descritores de forma por análise de variância univariada permutacional para otólitos de Coryphaena hippurus Linnaeus, 1758 capturados entre fevereiro de 2014 e outubro de 2016 na costa sudoeste do oceano Atlântico.

\begin{tabular}{lcccc}
\hline Fonte & df & Pseudo-F & P(perm) & Unique perms \\
\hline Anos & 2 & 27,054 & 0,0001 & 9946 \\
Res & 161 & & & \\
$\quad$ Total & 163 & & & 9,0001 \\
Área & 2 & 45,117 & 0,0001 & 9951 \\
Perímetro & 2 & 29,208 & 0,0104 & 9953 \\
Circularidade & 2 & 45,783 & 0,1698 & 9943 \\
Retangularidade & 2 & 1,825 & 0,0001 & 9952 \\
Excentricidade & 2 & 12,574 & 0,7405 & 9955 \\
Dimensão fractal & 2 & 0,3072 & 9940 \\
\hline
\end{tabular}

A ADL aplicada aos descritores de forma confirmou o padrão observado pela PERMANOVA, onde primeiro eixo (DL1) explicou $79,1 \%$ da variação, enquanto o eixo 2 (DL2) explicou 20,9\%. A matriz de classificação Jacknife gerou um valor de classificação de $52,2 \%$ para o ano de 2014; de $94,8 \%$ para o ano de 2015 e de $38,5 \%$ para o ano de 2016 (Fig. 2). Devido a melhor classificação do ano de 2015 associados a maior consistência de dados $(\mathrm{n}=115)$, comparações intra-anuais entre as áreas de pesca foram realizadas com a PERMANOVA, mas não apresentaram diferenças significativas (PERMANOVA, pseudo- $\mathrm{F}=1,83$; $\mathrm{p}=0,22$ ).

Análise de coeficientes de Fourier. A análise de agrupamento permitiu a identificação de quatro morfotipos predominantes de otólitos para a espécie na região de estudo (Fig. 3). Os morfotipos A e B apresentam as asas dorsal e ventral posteriores separadas, entretanto o morfotipo A apresenta o antirostro mais alongado (Fig.4). Os morfotipos $\mathrm{C}$ e $\mathrm{D}$ apresentam as asas dorsal e ventral posteriores fundidas, com o morfotipo D apresentando antirostro mais alongado (Fig. 4).

$\mathrm{Na}$ ACP gerada a partir da análise dos coeficientes de Fourier, o primeiro eixo (CP1) explicou 39,9\% da variação, enquanto o segundo eixo (CP2) explicou $24,2 \%$. Apesar dos 11 primeiros componentes terem sido significativos, a partir do quinto componente os autovalores se tornaram invariáveis, i.e., com variação irrelevante, enquanto os cinco primeiros eixos apresentam os principais aspectos morfológicos sujeitos a variação, que são os tamanhos do antirostro, comprimento das partes posteriores e nível de fusão das mesmas (Fig. 5).

Os dois primeiros eixos da ACP foram plotados por área de pesca, não apresentando um padrão de separação dos morfotipos (Fig. 6). Entretanto, quando os dois primeiros eixos foram plotados pelos diferentes morfotipos identificados na análise de agrupamento, foi encontrado um padrão de separação dos quatro grupos (Fig. 6).

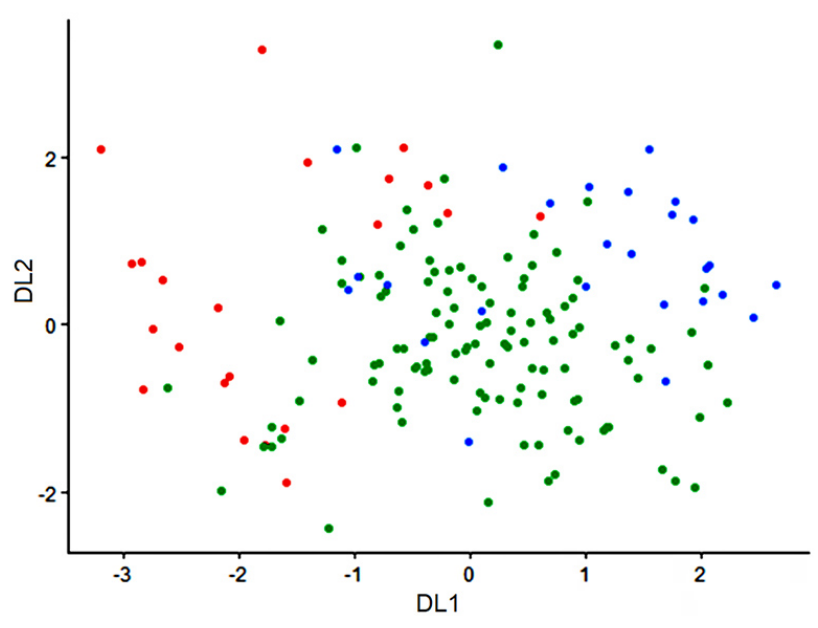

Fig. 2. Distribuição dos descritores de forma dos otólitos de Coryphaena hippurus Linnaeus, 1758 capturados em 2014 (•), 2015 (•) e 2016 (•) gerado a partir de uma análise discriminante linear. 


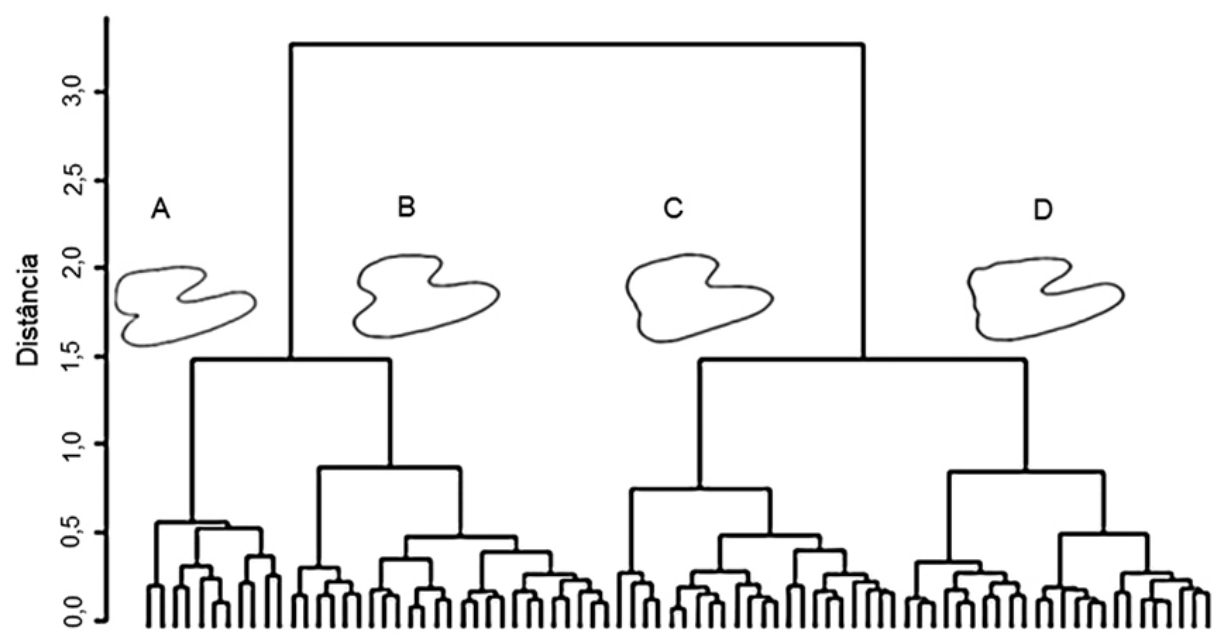

Fig. 3. Dendrograma da análise de agrupamento com base nas distâncias euclidianas das amplitudes das 30 primeiras harmônicas e contorno dos quatro morfotipos de otólitos sagitta de Coryphaena hippurus Linnaeus, 1758 registrados na costa sudoeste do Oceano Atlântico.

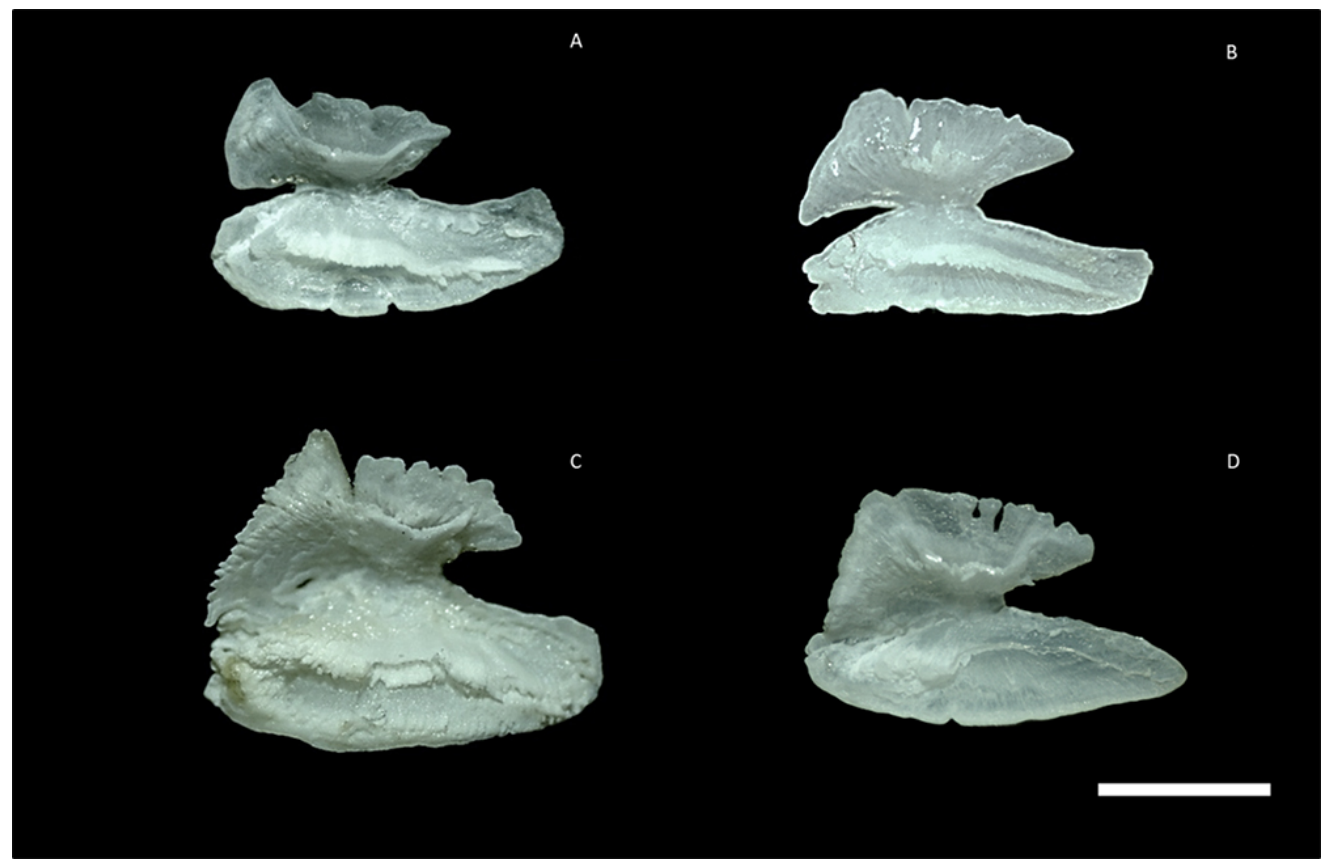

Fig. 4. Imagens digitais de exemplares de otólitos sagitta de Coryphaena hippurus Linnaeus, 1758 referentes aos morfotipos A, B, C e D encontrados para a área de estudo (escala $=1 \mathrm{~mm})$.

\section{DISCUSSÃO}

Os descritores de forma obtidos dos otólitos de C. hippurus se mostraram excelentes ferramentas preditoras do estrato desembarcado, i.e., temporalmente com diferenças significativas (variação interanual) e espacialmente sem distinção significativa entre as áreas de pesca. Por ser uma espécie oceânica migratória compartilhada por diferentes frotas e de rápido crescimento (LeSSA \& SANTANA, 2016), o dourado capturado no sudeste brasileiro encontra-se suscetível às variações das condicionantes oceanográficas desta região que, quando atingem grandes amplitudes (variações sazonais da ressurgência costeira), podem afetar o padrão de desenvolvimento dos indivíduos e consequentemente a morfologia dos otólitos em diversos recursos pelágicos (Palko et al., 1982; Olavo et al., 2005; Callicó-Fortunato et al., 2017).

As diferenças nos descritores de forma dos otólitos encontradas para os três ciclos anuais ressaltam como a dinâmica populacional do dourado pode variar temporalmente em uma região influenciada não só pelas condicionantes ambientais, mas também por outros fatores como reprodução, alimentação e interações bióticas (NUNES et al., 2015; RAJESH et al., 2016). Tais constatações podem ser corroboradas 


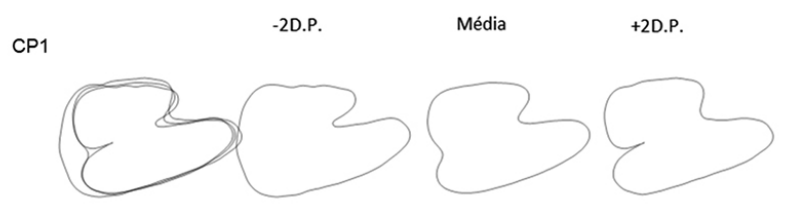

CP2

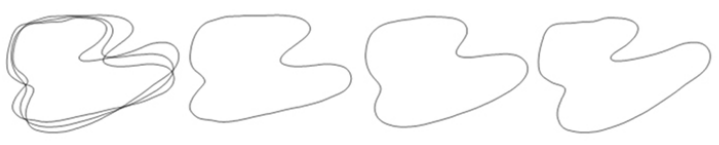

CP3

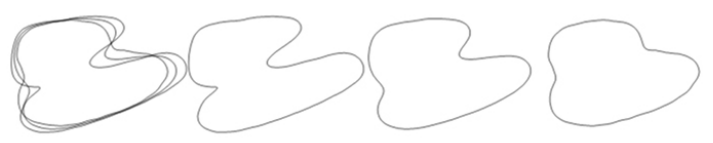

CP4

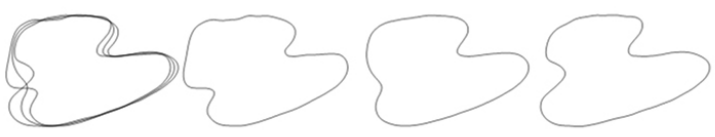

CP5

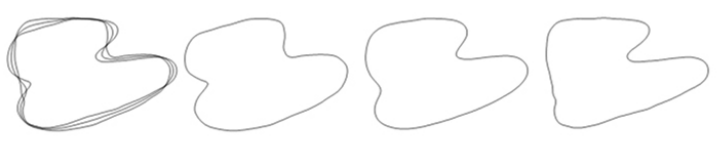

Fig. 5. Variações na forma dos otólitos sagitta de Coryphaena hippurus Linnaeus, 1758 explicada por cada componente principal (CP). A coluna da esquerda apresenta a sobreposição da média $\pm 2 \mathrm{X}$ o desvio padrão (D.P.) dos contornos das formas.

pelo postulado por PALKo et al. (1982) que afirmaram que as populações de dourado apresentam altas taxas de renovação podendo dobrar em até 15 meses, devido ao seu rápido crescimento. Resultados semelhantes são bem documentados na literatura, considerando a existência de certo grau de dependência entre a forma do otólito e variáveis oceanográficas. Estudos realizados por JónSDótTIR et al. (2006) relataram que, ao redor da Islândia, a variação no formato dos otólitos de Gadus morhua Linnaeus, 1758 entre dois anos consecutivos esteve associada às variações dos parâmetros ambientais locais, como temperatura e dinâmica das correntes oceanográficas. HüssY (2008) também observou, em experimento controlado, uma relação direta entre o desenvolvimento ontogenético de Gadus morhua e condicionantes ambientais capazes de influenciar na forma dos otólitos dos juvenis. MatTA et al. (2010) estudaram a correlação entre o crescimento dos otólitos de três espécies de linguado e variáveis climáticas como temperatura superficial do mar (TSM), cobertura de gelo, Oscilação Decadal do Pacífico e ENOS no Mar de Bering, registrando uma ação intensa da temperatura no padrão de crescimento das espécies na região devido à influência das oscilações climáticas.

De todas as variáveis de forma utilizadas no presente estudo, a dimensão fractal vem sendo amplamente utilizada pela comunidade científica para diferenciação de estoques através de otólitos devido a sua capacidade em distinguir com

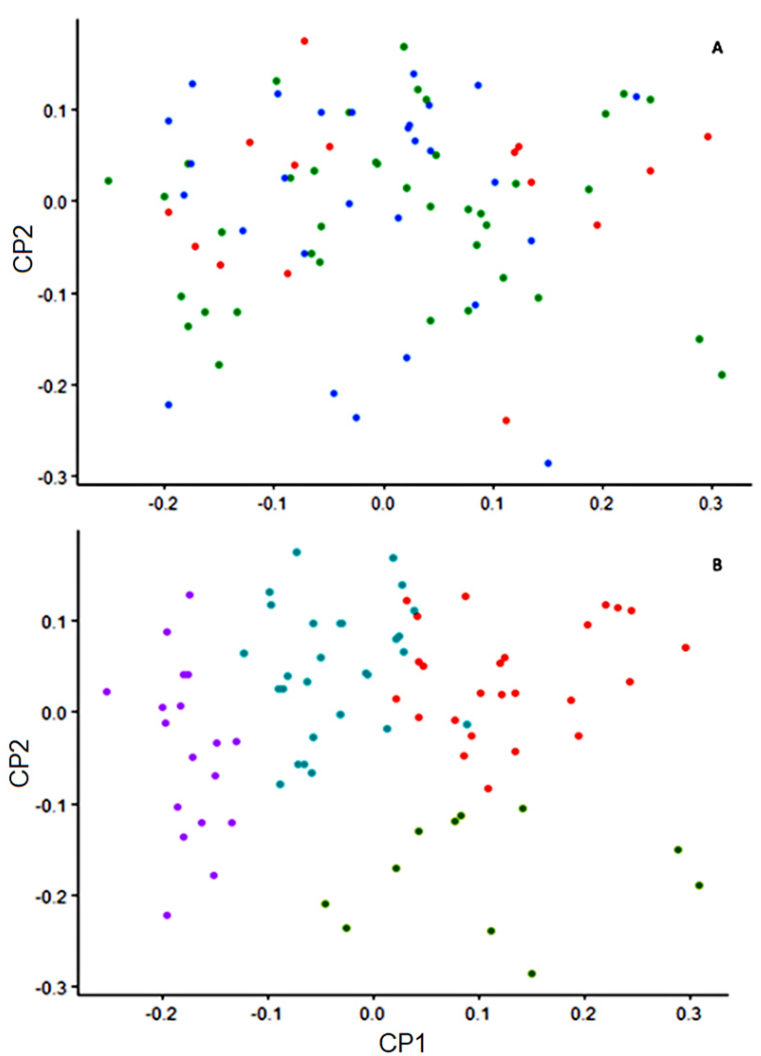

Fig. 6. Distribuição das amostras a partir da ordenação da análise de componentes principais das variáveis de Fourier: A, amostras ordenadas por áreas I (•), II (•) e III (•); B, amostras ordenadas por morfotipos A $(\bullet), \mathrm{B}(\bullet), \mathrm{C}(\bullet)$ e D $(\bullet)$.

eficiência a complexidade de formas em grupos amostrais distintos. Fractais são formas geométricas incapazes de ser classificada nos moldes da Geometria Euclidiana devido principalmente à sua auto semelhança em diferentes níveis de escala e sua complexidade infinita, onde a dimensão fractal representa seu nível de irregularidade (GULICK, 1992; BACKES \& BRUNO, 2005). Quanto maior a irregularidade de uma forma, maior é a sua dimensão fractal, sendo essa, portanto, uma característica que a torna uma ferramenta muito útil para a comparação de duas ou mais formas (COSTA \& CESAR JR, 2000). Ao estudarem esta mesma espécie na Região Nordeste do Brasil, DuARTE-Neto et al. (2008) observaram que a dimensão fractal foi o descritor mais importante na diferenciação dos dois estoques da espécie existentes na região. No presente estudo, ainda que diferenças significativas entre os ciclos anuais tenham sido encontradas para a maioria dos descritores de forma, a ausência de variação na dimensão fractal dos otólitos reforça a hipótese de um estoque misto, cujas variações temporais podem ser decorrentes das condicionantes ambientais, alimentação e condição dos indivíduos (CAMPana \& CASSELMan, 1993; Rossi-WongTSCHOWSKI, 2015).

Embora a complexidade de forma do otólito de $C$. hipurrus seja um fator robusto para a diferenciação dos estoques, não foram identificadas diferenças significativas para separação dos morfotipos entre as áreas de pesca. 
Uma das hipóteses que pode suportar a presença dos quatro morfotipos está relacionada aos hábitos alimentares da espécie, i.e., um predador de topo com uma dieta baseada, predominantemente, no consumo de espécies epipelágicas com ampla ocorrência, associada à elevada produtividade na região - ressurgência de Cabo Frio (Valentin \& CoutinHo, 1990). Alguns autores têm reportado diferenças ontogenéticas e sazonais na dieta da espécie, estando tais variações associadas a distintos fatores (OXENFORD \& HUNTE, 1999; BREWTon et al., 2016), entre os quais se destaca a habilidade natatória da espécie, que permite a mesma explorar presas diversas em áreas distintas inclusive mais distantes da costa (MassuTí et al., 1998). Além da alimentação outros atributos bionômicos, como o crescimento e a reprodução, também podem responder pelas conformações dos otólitos (Rossi-WongTschOWSKI, 2015). Além disso, tais parâmetros de história de vida são reconhecidamente influenciados pelas condicionantes ambientais exercendo distintos graus de influência no desenvolvimento da forma dos otólitos (Richter et al., 2000; Gagliano \& MCCORMick, 2004; CARDinale et al., 2004). SALVANES et al. (2004) apontaram a distinção de duas subpopulações de Gadus morhua na Noruega, onde os peixes de maiores latitudes apresentaram menores tamanhos, pesos, taxas de crescimento e fator de condição do que os de latitudes inferiores, mostrando como diferentes condicionantes oceanográficas podem influenciar na história de vida de uma espécie. LATTUCA et al. (2015) observaram a relação entre o fator de condição e atributos como idade, crescimento, dieta e reprodução na diferenciação de forma dos otólitos de Odontesthes nigricans (Richardson, 1848) em duas áreas no extremo sul da Argentina. Todas estas evidências mostram que os padrões de distribuição e abundância de $C$. hippurus estão sujeitos a dinâmica oceanográfica. De acordo com FARREL et al. (2014), o dourado apresenta uma preferência por águas com elevada TSM e baixas concentrações de clorofila-a (CL), condições essas recorrentes na área de estudo devido ao fenômeno de ressurgência sazonal observado nas áreas de captura em função dos deslocamentos da corrente do Brasil. Neste contexto, as diferenças observadas nos fatores de forma podem estar relacionadas às condições dos indivíduos analisados (amplitude de CF), concordando que os otólitos são espécie-específicos moldados por fatores intrínsecos e extrínsecos locais, i.e., estrutura em tamanho/condição individual e variáveis oceanográficas, respectivamente.

Os parâmetros ambientais da PCSE apresentam um padrão de variação sazonal em que os valores médios anuais de TSM e CL são constantes, com oscilações regulares em certos períodos do ano e apresentando picos de TSM no verão e de CL no inverno (GAETA \& BRANDini, 2006). Entretanto, valores fora do padrão de oscilação comum dos parâmetros oceanográficos são registrados em certos períodos, correspondendo à ocorrência dos ENOS. Seus efeitos na PCSE são amplamente conhecidos, cuja influência abrange variações de temperatura, pressão atmosférica ao nível do mar e outras variáveis de superfície (TSM e CL), além de amplificar a produtividade primária após El Niños intensos e diminuir em caso de El Niños moderados ou $\mathrm{La}$ Niñas (Aceituno, 1988; PAes \& Moraes, 2007). Desta forma, os registros destes fenômenos também podem responder pelas variações temporais na história de vida e nos otólitos de C. hippurus da costa sudoeste do Atlântico.

A ausência de diferenças significativas entre as áreas de pesca, tanto para as análises dos descritores de forma quanto para os coeficientes de Fourier para o ano de 2015, consolida a hipótese da existência de um estoque misto de dourado capturado na região sudeste do Brasil. Resultados semelhantes foram observados por MAHÉ et al. (2016), para o peixe-espada Xiphias gladius Linnaeus, 1758 no Oceano Índico, onde os autores não encontraram diferenças significativas entre as áreas amostradas, corroborando para a consolidação de um único estoque na região. Através dos coeficientes de Fourier estimados foram identificados quatro morfotipos predominantes para $C$. hippurus na região de estudo. Enquanto Duarte-Neto el al. (2008), ao compararem populações de C. hippurus das correntes do Brasil e do norte do Brasil através de capturas na costa da região Nordeste da costa brasileira, encontraram três morfotipos de otólito dominantes, os quais não apresentaram distinção geográfica apesar de validada a separação dos estoques através da dimensão fractal. Esses três morfotipos, descritos por DUARTE-NETO et al. (2008) como I = asa dorsal e ventral de tamanho equivalente e parte posterior separada, $\mathrm{II}=$ asa dorsal mais curta que a ventral e parte posterior fundida e III = asa dorsal mais curta que a ventral e parte posterior separada, são equivalentes aos morfotipos $\mathrm{A}, \mathrm{C}$ e $\mathrm{B}$, respectivamente, encontrados no presente estudo, embora tenhamos encontrado um morfotipo dominante a mais, D, aninhado ao morfotipo $\mathrm{C}$. Tais diferenças na quantidade de morfotipos dominantes podem ser decorrentes tanto de variações espaciais quanto temporais, permitindo a inferência de duas hipóteses. A primeira na qual a distância geográfica e fatores ambientais clinais associados estariam discriminando a população de dourado que utiliza a corrente do Brasil (rasa, quente e salina) como rota migratória de dois estoques, sendo um localizado na região Nordeste e outro na Sudeste, com algumas áreas de misturas de ambos os estoques. Tal suposição é fundamentada em um processo de seleção balanceado que ocorre ao longo de diferentes regiões com características ambientais e oceanográficas distintas, i.e., ao longo da rota migratória do dourado, resultando em uma seleção-k (GAULDIE \& CRAMPTON, 2002). Este processo consiste no aumento da eficiência de utilização dos recursos ambientais em espécies cujas populações são limitadas principalmente pela capacidade suporte do ambiente (PIANKA, 1970). Assim, espécies de peixes pelágicos migradores são levadas a explorarem amplas áreas beneficiando-se de diferentes nichos. Não obstante, AHLStrom (1957) já havia reportado que tunídeos e clupeídeos com fenótipos específicos, ao se deslocarem entre regiões distintas em diversas escalas de tempo, são capazes de gerar descendentes com características fenotípicas distintas induzidas pelas diferentes condições ambientais. No entanto, as diferenças de formas dos otólitos entre áreas podem ser mais difíceis de 
serem observadas em espécies migratórias devido à amplitude geográfica que são capazes de percorrer, o que contribui para ocorrência de estoques mistos, além da influência dos fatores ambientais sobre um mesmo grupo de indivíduos. A segunda hipótese é que a população de $C$. hippurus que utiliza a corrente do Brasil corresponderia a um único estoque, mesmo com os otólitos apresentado variações de morfotipos. Tal suposição se justificaria pelo processo de seleção por equilíbrio temporal baseada em um polimorfismo genético persistente na população, onde diferentes morfotipos são dominantes alternadamente em um determinado intervalo de tempo (GAuldie \& Crampton, 2002), influenciados por fatores capazes de intervir no genótipo e fenótipo dos indivíduos, devido a efeitos de seleção natural ou estocástica.

Adicionalmente, BONNER et al. (2017) realizaram estudos genéticos sobre dois morfotipos de dourados desembarcados e comercializados ao largo da costa do Rio de Janeiro e concluíram que se trata de um estoque misto, cuja hipótese de seleção natural é discutida como possível explicação para seus resultados. Logo, não refutamos nenhuma das hipóteses levantadas uma vez que as evidências do presente estudo e dos resultados observados por DUARTENETO et al. (2008) podem ser corroboradas pela segunda hipótese. Já a primeira hipótese se sustenta pela existência desta área de ressurgência, cujas mudanças sazonais podem ou não ser intensificadas em função de eventos climáticos de dimensões globais, ocasionando alterações em populações que cruzam e/ou utilizam estas áreas em algum momento do seu ciclo de vida (GAETA \& Brandini, 2006; PAes \& Moraes, 2007). Segundo FARRELl et al. (2014), variações das condicionantes ambientais estão fortemente relacionadas aos padrões de distribuição da espécie, além de estarem correlacionadas com o nível de produtividade dos ambientes marinhos, acarretando em maior disponibilidade de alimento para a espécie. Fatos estes documentados na literatura devido a seu hábito de espécie cosmopolita com diferentes parâmetros de história de vida registrados em diversas localidades do mundo (PALKo et al., 1982; OXENFORD, 1999; GATT et al., 2015; LesSa \& SANTANA, 2016). Portanto, podemos concluir que nossos resultados concordam parcialmente com ambas as hipóteses, uma vez que tais padrões de desenvolvimento dos otólitos descritos na literatura podem ser aplicados/ confrontados à espécie alvo devido a seus atributos de história de vida, bem como a suas correlações com as variáveis ambientais. Assim, admitimos a existência de um estoque misto uma vez que as áreas de pesca são homogêneas quanto à presença dos morfotipos registrados, além de corroborar o estudo genético produzido na região, cujos componentes de variação genotípica foram idênticos entre os morfotipos avaliados. No entanto, para elucidar completamente a questão em torno da dinâmica dos possíveis estoques de dourado da costa do Brasil, seriam necessários mais estudos abordando morfologia/morfometria de otólitos ao longo de toda sua extensão geográfica e seus padrões de variação temporal, aliados a estudos genéticos modernos e/ou de marcação e recaptura.
Agradecimentos. Ao Conselho Nacional de Desenvolvimento Científico e Tecnológico (CNPq), à Coordenação de Aperfeiçoamento de Pessoal de Nível Superior (CAPES) e à Fundação Carlos Chagas Filho de Amparo à Pesquisa do Estado do Rio de Janeiro (FAPERJ) pelo apoio financeiro. À Universidade Federal Fluminense (UFF) e à Fundação Instituto de Pesca do Estado do Rio de Janeiro (FIPERJ) pelo apoio logístico.

\section{REFERÊNCIAS}

Aceituno, P. 1988. On the functioning of the Southern Oscillation in the South American sector. Part I: Surface climate. Monthly Weather Review 116(3):505-524.

Ahlstrom, E. H. 1957. A review of recent studies of subpopulations of Pacific fishes. In: MARR, J. C. ed. Contributions to the study of subpopulations of fishes. Washington D. C., Special Scientific Report Fisheries, p. 44-73.

Anderson, M.; Gorley, R. \& Clarke, K. P. 2008. PRIMER: guide to software and statistical methods. Plymouth, PRIMER-E. 218p.

Ávila-DA-Silva, A. O. \& VAZ-Dos-Santos, A. M. 2000. Análise das capturas de atuns e afins pelos métodos de vara e isca viva e corrico realizadas pelo N/P Malacostraca de 1980 a 1991. Boletim do Instituto de Pesca 26(2):211-221.

BACKes, A. R. \& BRUno, O. M. 2005. Técnicas de estimativa da dimensão fractal: um estudo comparativo. INFOCOMP 4(3):50-58.

Baumann, H.; Gröhsler, T.; Kornilovs, G.; Makarchouk, A.; Feldmann, V. \& Temming, A. 2006. Temperature-induced regional and temporal growth differences in Baltic young-of-the-year sprat Sprattus sprattus. Marine Ecology Progress Series 317:225-236.

BegG, G. A. \& Waldman, J. R. 1999. An holistic approach to fish stock identification. Fisheries Research 43(1-3):35-44.

Bonner, A.; Duarte, M. R.; Souza, R. C.; Monteiro-Neto, C. \& Silva, E. P. 2017. Taxonomic status of two morphotypes of Coryphaena hippurus (Perciformes: Coryphaenidae). Neotropical Ichthyology 15(1):e160102.

Brewton, R. A.; Ajemian, M. J.; Young, P. C. \& Stunz, G. W. 2016. Feeding Ecology of Dolphinfish in the Western Gulf of Mexico. Transactions of the American Fisheries Society 145(4):839-853.

Callicó-Fortunato, R.; DurÀ, V. B.; GonZÁlez-Castro, M. \& Volpedo, A. 2017. Morphological and morphometric changes of sagitta otoliths related to fish growth in three Mugilidae species. Journal of Applied Ichthyology 33(6): 1137-1145.

Campana, S. E. 1999. Chemistry and composition of fish otoliths: pathways, mechanisms and applications. Marine Ecology Progress Series 188:263-297.

Campana, S. E. \& Casselman, J. M. 1993. Stock discrimination using otolith shape analysis. Canadian Journal of Fisheries and Aquatic Sciences 50(5):1062-1083.

Campana, S. E.; Chouinard, G. A.; Hanson, J. M.; Frechet, A. \& Brattey, J. 2000. Otolith elemental fingerprints as biological tracers of fish stocks. Fisheries Research 46(1-3):343-357.

Cardinale, M.; Doering-Arjes, P.; Kastowsky, M. \& Mosegaard, H. 2004. Effects of sex, stock, and environment on the shape of known-age Atlantic cod (Gadus morhua) otoliths. Canadian Journal of Fisheries and Aquatic Sciences 61(2):158-167.

Castonguay, M.; Simard, P. \& Gagnon, P. 1991. Usefulness of Fourier analysis of otolith shape for Atlantic mackerel (Scomber scombrus) stock discrimination. Canadian Journal of Fisheries and Aquatic Sciences 48(2): 296-302.

CAstro, B. M. \& LeE, T. N. 1995. Wind-forced sea level variability on the southeast Brazilian shelf. Journal of Geophysical Research: Oceans 100(C8):16045-16056.

Castro, B. M. \& Miranda, L. B. 1998. Physical oceanography of the Western Atlantic Continental shelf located between $4^{\circ} \mathrm{N}$ and $34^{\circ} \mathrm{S}$. In: Robinson, A. R. \& BRInK, K. H. eds. The Sea - The Global Coastal Ocean-Regional Studies and Synthesis. New York, John Wiley \& Sons, p. 209-251.

Clarke, K. R. \& Gorley, R. N. 2006. Primer. Plymouth, PRIMER-e. 20p. Costa, L. F \& Cesar JR, R. M. 2000. Shape Analysis and Classification: Theory and Practice. Boca Raton, CRC Press. 680p. 
COSTA, L. F \& Cesar JR, R. M. 2010. Shape analysis and classification. New York, CRC Press. 659p.

Dallagnolo, R. \& Andrade, H. A. 2008. Observações a respeito da pescaria sazonal de dourado (Coryphaena hippurus) com espinhel-desuperfície no sul do Brasil. Boletim do Instituto de Pesca 34(2):331335.

Diaz, G.; Cappai, C.; Setzu, M. D.; Sirigu, S. \& Diana, A. 1997. Elliptic Fourier descriptors of cell and nuclear shapes. In: Lestrel, P. E. ed. Fourier descriptors and their applications in biology. New York, Cambridge University Press, p. 307-321.

Duarte-Neto, P.; Lessa, R.; Stosic, B. \& Morize, E. 2008. The use of sagittal otoliths in discriminating stocks of common dolphinfish (Coryphaena hippurus) off northeastern Brazil using multishape descriptors. ICES Journal of Marine Science 65(7):1144-1152.

Farrell, E. R.; Boustany, A. M.; Halpin, P. N. \& Hammond, D. L. 2014. Dolphinfish (Coryphaena hippurus) distribution in relation to biophysical ocean conditions in the northwest Atlantic. Fisheries Research 151:177-190.

Gaeta, S. A. \& Brandini, F. P. 2006. Produção primária do fitoplâncton na região entre o Cabo de São Tomé (RJ) e o Chuí (RS). In: RossiWongtschowsKi, C. L. D. B. \& MaduReira, L. S. eds. O ambiente oceanográfico da plataforma continental e do talude na região sudeste-sul do Brasil. São Paulo, EDUSP, p. 219-264.

GaGLiAnO, M. \& MCCORMICK, M. I. 2004. Feeding history influences otolith shape in tropical fish. Marine Ecology Progress Series 278:291-296.

GatT, M.; DimeCH, M. \& SCHEMBRi, P. J. 2015. Age, growth and reproduction of Coryphaena hippurus (Linnaeus, 1758) in Maltese waters, Central Mediterranean. Mediterranean Marine Science 16(2):334-345.

GAULDIE, R. W. \& CRAMPTON, J. S. 2002. An eco-morphological explanation of individual variability in the shape of the fish otolith: comparison of the otolith of Hoplostethus atlanticus with other species by depth. Journal of Fish Biology 60(5):1204-1221.

Gulick, D. 1992. Encounters with Chaos. New York, McGraw-Hill College. $387 \mathrm{p}$

Hüssy, K. 2008. Otolith shape in juvenile cod (Gadus morhua): Ontogenetic and environmental effects. Journal of Experimental Marine Biology and Ecology 364(1):35-41.

IWATA, H. \& UKAI, Y. 2002. SHAPE: a computer program package for quantitative evaluation of biological shapes based on elliptic Fourier descriptors. Journal of Heredity 93(5):384-385.

JónsdótTiR, I. G.; CAMPana, S. E. \& MarteinsDottir, G. 2006. Otolith shape and temporal stability of spawning groups of Icelandic cod ( Gadus morhua L.). ICES Journal of Marine Science 63(8):1501-1512.

KuHL, F. P. \& Giardina, C. R. 1982. Elliptic Fourier features of a closed contour. Computer Graphics and Image Processing 18:236-258.

Lattuca, M. E.; Lozano, I. E.; Brown, D. R.; Renzi, M. \& Luizon, C. A. 2015. Natural growth, otolith shape and diet analyses of Odontesthes nigricans Richardson (Atherinopsidae) from southern Patagonia. Estuarine, Coastal and Shelf Science 166:105-114.

Lessa, R. \& Santana, F. M. 2016. Growth of the dolphinfish Coryphaena hippurus from north-eastern Brazil with an appraisal of the efficacy of scales and otoliths for ageing. Journal of Fish Biology 89(1):977-989.

Lleonart, J.; Salat, J. \& TORRES, G. J. 2000. Removing allometric effects of body size in morphological analysis. Journal of Theoretical Biology 205(1):85-93.

Lorenzzetti, J. A. \& Gaeta, S. A. 1996. The Cape Frio Upwelling effect over the South Brazil Bigth northern sector shelf waters: a study using AVHRR images.International Society of Photogrametry and Remote Sensing. Vienna, $\mathbf{1 8}^{\circ}$ ISPRS Congress, p. 448-453.

Mahe, K.; Evano, H.; Mille, T.; Muths, D. \& BourJea, J. 2016. Otolith shape as a valuable tool to evaluate the stock structure of swordfish Xiphias gladius in the Indian Ocean. African Journal of Marine Science 38(4): 457-464.

Massutí, E.; Deudero, S.; SÁNChez, P. \& Morales-Nin, B. 1998. Diet and feeding of dolphin (Coryphaena hippurus) in western Mediterranean waters. Bulletin of Marine Science 63(2):329-341.

Matta, M. E.; Black, B. A. \& Wilderbuer, T. K. 2010. Climate-driven synchrony in otolith growth-increment chronologies for three Bering Sea flatfish species. Marine Ecology Progress Series 413:137-145.

Mohriak, W. U.; Nóbrega, M.; Odegard, M. E.; Gomes, B. S. \& Dickson, W. G. 2010. Geological and geophysical interpretation of the Rio Grande
Rise, south-eastern Brazilian margin: extensional tectonics and rifting of continental and oceanic crusts. Petroleum Geoscience 16:231-245.

Monteiro, L. R.; Di Beneditto, A. P. M.; Guillermo, L. H. \& Rivera, L. A. 2005. Allometric changes and shape differentiation of sagitta otoliths in sciaenid fishes. Fisheries Research 74(1-3):288-299.

Monteiro, L. R. \& Reis, S. F. D. 1999. Princípios de morfometria geométrica. Ribeirão Preto, Holos. 198p.

Muene, D. \& Garcez, D. S. 2005. The Brazilian continental shelf and its relation with the coastal zone and fishing. Mercator 4(8):69-88.

Nunes, J. A. C. C.; Freitas, R. H. A.; Reis-Filho, J. A.; Loiola, M. \& SAMAIO, C. L. S. 2015. Feeding behavior of the common dolphinfish Coryphaena hippurus: older fish use more complex foraging strategies. Journal of the Marine Biological Association of the United Kingdom 95(6): 1277-1284.

Olavo, G.; Costa, P. A. S. \& Martins, A. S. 2005. Prospecção de grandes peixes pelágicos na região central da ZEE brasileira entre o Rio RealBA e o cabo de São Tomé-RJ. In: Costa, P. A. S.; Martins, A. S. \& Olavo, G. eds. Pesca e potenciais de exploração de recursos vivos na região central da Zona Econômica Exclusiva brasileira. Rio de Janeiro. Museu Nacional, p. 167-202.

OXENFORD, H. A. 1999. Biology of the dolphinfish (Coryphaena hippurus) in the western central Atlantic: a review. Scientia Marina 63:277-301.

OxenFord, H. A. \& Hunte, W. 1999. Feeding habits of the dolphinfish (Coryphaena hippurus) in the eastern Caribbean. Scientia Marina 63(34):303-315.

Paes, E. T. \& Moraes, L. E. S. 2007. A new hypothesis on the influence of the El Niño/La Niña upon the biological productivity, ecology and fisheries of the Southern Brazilian Bight. Pan-American Journal of Aquatic Sciences 2(2):94-102.

Palko, B. J.; Beardsley, G. L. \& Richards, W. J. 1982. Synopsis of the biological data on dolphin fishes, Coryphaena hippurus Linnaeus and Coryphaena equiselis Linnaeus. Highlands, NOAA Technical Report NMFS. 28p.

Pauly, D. \& Christensen, V. 1995. Primary production required to sustain global fisheries. Nature 374:255-257.

Pianka, E. R. 1970. On r-and K-selection. The American Naturalist 104(940):592-597.

Popper, A. N. \& Lu, Z. 2000. Structure-function relationships in fish otolith organs. Fisheries Research 46(1-3):15-25.

Rajesh, K. M.; Rohit, P. \& Abdussamad, E. M. 2016. Fishery diet composition and reproductive biology of the dolphinfish Coryphaena hippurus (Linnaeus, 1758) off Karnataka, south-west coast of India. Indian Journal of Fisheries 63(4):35-40.

ReIs, E. 1997. Estatística multivariada aplicada. Lisboa, Sílabo. 343p.

Richter, H.; Luckstadt, C.; Focken, U. \& Becker, K. 2000. An improved procedure to assess fish condition on the basis of length-weight relationships. Archive of Fishery and Marine Research 48(3):255-264.

Rondon, A. S.; VAZ-Dos-SAntos, A. M. \& Rossi-WongtschowsKi, C. L. D. B. 2014. Morfologia e biometria dos otólitos de Beryx splendens e Hoplostethus occidentalis (Beryciformes) no Atlântico Sudoeste. Boletim do Instituto de Pesca 40(2):195-206.

Rossi-Wongtschowski, C. L. D. B. 2015. Morfologia de otólitos. In: Volpedo, A. V. \& VAZ-Dos-Santos, A. M. eds. Métodos de estudo com otólitos: princípios e aplicações. Buenos Aires, CAFP-BAPIESCI, p. 29-58.

Russ, J. C. 1990. Image processing. In: Russ, J. C. ed. Computer-Assisted Microscopy. Boston, Springer, p. 33-69.

Ryther, J. H. 1969. Photosynthesis and fish production in the sea. The production of organic matter and its conversion to higher forms of life vary throughout the world ocean. Science 166:72-76.

Salvanes, A. G. V.; SkJeraAsen, J. E. \& Nilsen, T. 2004. Sub-populations of coastal cod with different behaviour and life-history strategies. Marine Ecology Progress Series 267:241-251.

Santos, R. S.; Azevedo, M. C. C.; Albuquerque, C. Q. \& Araújo, F. G. 2017. Different sagitta otolith morphotypes for the whitemouth croaker Micropogonias furnieri in the Southwestern Atlantic coast. Fisheries Research 195:222-229.

Silva, N. M. L.; Valentin, J. L. \& Bastos, C. T. B. T. 1988. O microfitoplâncton das águas costeiras do litoral fluminense (Estado do Rio de Janeiro): lista de espécies e aspectos ecológicos. Boletim do Instituto Oceanográfico 36(1/2):1-16. 
Soeth, M.; Spach, H. L.; Daros, F. A.; Adelir-Alves, J.; Almeida, A. C. O. \& Correia, A. T. 2019. Stock structure of Atlantic spadefish Chaetodipterus faber from Southwest Atlantic Ocean inferred from otolith elemental and shape signatures. Fisheries Research 211:81-90.

SPARRE, P. \& VenEMA, S. 1997. Introdução à avaliação de mananciais de peixes tropicais. FAO Documento Técnico Sobre as Pescas 306(1):1-404.

Stevenson, M. R.; Dias-Brito, D.; Stech, J. L. \& Kampel, M. 1998. How do cold water biota arrive in a tropical bay near Rio de Janeiro, Brazil? Continental Shelf Research 18(13):1595-1612.

Tuset, V. M.; Lozano, I. J.; González, J. A.; Pertusa, J. F. \& GarcíaDíAZ, M. M. 2003. Shape indices to identify regional differences in otolith morphology of comber, Serranus cabrilla (L., 1758). Journal of Applied Ichthyology 19(2):88-93.

VALENTIN, J. L. \& CoutinHO, R. 1990. Modelling maximum chlorophyll in the Cabo Frio (Brazil) upwelling: A preliminary approach. Ecological Modelling 52:103-113.

VaZ-Dos-SAntos, A. M.; Rossi-Wongtschowski, C. L. D. B. \& Figueiredo, J. L. 2007. Recursos pesqueiros compartilhados: bioecologia, manejo e aspectos aplicados no Brasil. Boletim do Instituto de Pesca 33(2):273292.

Venables, W. N. \& Ripley. B. D. 2002. Modern applied statistics with S. New York, Springer Science \& Business Media, v.4. 498p. 